\title{
Aprendizaje a distancia del análisis sensorial de vinos
}

\author{
Juan Gómez Beníteza , Cristina Lasanta Melero y Manuel A. Cobo Heredia \\ Departamento de Ingeniería Química y Tecnología de Alimentos, Facultad de Ciencias, Universidad de Cádiz, \\ 11510 Puerto Real, España
}

\begin{abstract}
Resumen. En esta comunicación se presentan los resultados de un proyecto de innovación educativa en análisis sensorial de vinos desarrollado en la Universidad de Cádiz (España). El sistema utilizado se basa en la cuantificación de 37 atributos sensoriales del vino relacionados con el aspecto visual, aroma, sabor y apreciación global en una escala de 0 (inexistente), 1 (perceptible), 2 (considerable) y 3 (muy intenso), empleando las herramientas del software libre Moodle de educación a distancia. Los vinos elegidos son catados previamente por los profesores y sus resultados son cargados en el sistema como un examen tipo test de múltiple respuesta. Los alumnos catan en sus domicilios las mismas referencias de vino y cargan sus resultados en el programa al que se accede por Internet. El aprendizaje del alumno se mide en base a la diferencia de su evaluación con la de los profesores. Se organizaron tres cursos no presenciales entre los años 2012 y 2014 con un total de 106 alumnos. Para estudiar los resultados se ha realizado un análisis estadístico utilizando el test Kruskal-Wallis para las variables no paramétricas y ANOVA para las paramétricas. Como resultado se ha comprobado que los alumnos mostraron un aprendizaje destacable y creciente durante el curso. Los análisis estadísticos han mostrado además que las mujeres han obtenido calificaciones significativamente mejores que las de los hombres, que los catadores con experiencia media han tenido mejores calificaciones que los de experiencia amplia y que los vinos tintos de crianza han sido los mejores calificados. Además, en una encuesta anónima realizada al final de los cursos, los alumnos mostraron un elevado grado de satisfacción valorado en 8.67/10.
\end{abstract}

\section{Introducción}

Se entiende como aprendizaje a distancia o e-learning el desarrollo del proceso de formación (reglada o no reglada), basado en el uso de las tecnologías de la información y las telecomunicaciones (TIC), que posibilitan un aprendizaje interactivo, flexible y accesible a cualquier receptor potencial, aprovechando sobre todo los medios que ofrece la red Internet [1]. Actualmente, el e-learning se emplea en educación superior en ámbitos tan diversos como la medicina [2], química [3], o ingeniería [4]. En relación a la utilización del e-learning en Enología, existen innumerables cursos de enología y de cata de vino a distancia, incluyendo algunos ofrecidos por prestigiosas universidades [5,6]. Por otra parte, el análisis sensorial de vinos suele ser bastante subjetivo y no suele ser habitual utilizar las técnicas de análisis sensorial y análisis estadístico desarrolladas para otros alimentos. Por ello, estos cursos acostumbran a limitarse a unos contenidos cargados en el servidor en los que se enseñan las técnicas de cata y se describen los vinos elegidos. Estos contenidos son descargados y recibidos por el alumno de una forma unidireccional [7]. Como consecuencia, no se tiene constancia de que los cursos de cata de vino incorporen técnicas de evaluación objetiva de los vinos que se catan con tratamiento estadístico de los resultados y de medida del aprendizaje de los alumnos que los reciben. Para superar estas deficiencias, este proyecto incorpora técnicas objetivas de análisis sensorial aplicadas a vinos, y utiliza herramientas de software específico de educación

\footnotetext{
a Autor de correspondencia: juangomez. benitez@uca.es
}

a distancia que además permiten obtener y analizar los resultados obtenidos por los alumnos. Para asegurar la fiabilidad estadística de los resultados se aplicaron diferentes técnicas de análisis estadístico, dependiendo de la finalidad deseada. En esta comunicación se expone la experiencia adquirida en el diseño, desarrollo y aplicación de un sistema de enseñanza-aprendizaje a distancia de análisis sensorial de vinos a nivel de iniciación, como un proyecto de innovación educativa desarrollado y aplicado en la Universidad de Cádiz (España) durante los años 2012-2014.

\section{Materiales y métodos}

\subsection{Estructura del curso}

El curso está dividido en tres bloques que se van abriendo sucesivamente a medida que el curso transcurre. El primero es de introducción y teoría del análisis sensorial, el segundo de ejercicios de adiestramiento de los sentidos, y el tercero propiamente de análisis sensorial de vinos comerciales. Los ejercicios de adiestramiento de la vista se realizan con disoluciones preparadas con colorantes alimentarios, los del olfato con una colección de aromas que se envía a los alumnos, así como disoluciones de diferentes materias (frutos, especies... etc.), cuyas recetas son facilitadas a los alumnos. Por último, el adiestramiento del gusto y del tacto bucal se realiza con disoluciones de sustancias fáciles de preparar a escala domiciliar. Además, el curso cuenta con una carta de colores preparada al efecto que se envía a los alumnos junto con los aromas. Esta carta de colores presenta todos los posibles colores

This is an Open Access article distributed under the terms of the Creative Commons Attribution License 4.0, which permits unrestricted use, distribution, and reproduction in any medium, provided the original work is properly cited. 
de los vinos y sus denominaciones y constituye un elemento de ayuda muy valioso para evaluar el color de los vinos. El curso cuenta con numerosas clases grabadas al efecto y una colección de audiovisuales de vinos de diferentes regiones españolas. Aunque la primera edición se desarrolló totalmente a distancia, se observó la conveniencia de realizar alguna sesión presencial. Por ello, en la segunda y tercera ediciones se realizaron dos sesiones presenciales, una al principio del curso para tomar el primer contacto con la técnica de análisis sensorial utilizada y otra al final para resolver dudas que fueran surgiendo durante el curso. Estas sesiones se grabaron y se facilitaron a todos los alumnos, además de que pudieron ser seguidas en directo a través del propio curso para los alumnos que decidieron obtener los vinos por su cuenta y realizarlas en su domicilio. La duración total del curso fue de tres meses. Además, se realizaban tutorías electrónicas por videoconferencia de forma periódica, normalmente una vez por semana.

\subsection{Muestras de vino}

La selección de los vinos utilizados se realizó con criterios de representatividad en su tipo, facilidad de compra en toda España y precio asequible. Se eligieron 60 vinos de diferentes tipos, facilitándose esa relación a los alumnos que debían adquirir en el mercado y hacer el análisis sensorial de las mismas referencias de al menos 14 vinos, siendo 3 vinos blancos, 2 vinos rosados, 2 vinos tintos jóvenes, 2 vinos tintos roble/crianza, 2 vinos tintos reserva/gran reserva y 3 vinos generosos, para poder optar al certificado de aprovechamiento en el curso.

\subsection{Profesores del curso y panel de cata}

El panel de cata está formado por 5 profesores de análisis sensorial entrenado según los criterios de las normas ISO 8586:2012 [8].

\subsection{Análisis sensorial de los vinos}

Se utilizó la técnica de análisis sensorial descriptivo cuantitativo conocida como Fixed-Choice Profiling que utiliza una lista cerrada de descriptores con una puntuación determinada para cada uno [9-12,14]. Los descriptores se eligieron por consenso y siguiendo los siguientes criterios

- Relevancia: estar presente frecuentemente en los vinos.

- Claridad: debe estar bien definido y no ser ambiguo.

- Independencia: no debe ser sinónimo ni redundante con otros descriptores.

- Discriminación: usar términos con amplio rango de valoración y evitando términos triviales.

La relación de descriptores se presenta en la Tabla 1.

Como se puede observar, se utilizaron 9 descriptores para el color y la transparencia, 20 para el olor, 7 para el sabor y 1 para la calidad global, en total 37 descriptores. Aunque para la calidad de los tratamientos estadísticos posteriores se aconseja que la evaluación de los descriptores se realice al menos de 0 a 5, teniendo en cuenta el nivel iniciación de los cursos la puntuación de cada descriptor se realizó en una escala de 0 (inexistente),
Tabla 1. Relación de descriptores utilizados.

\begin{tabular}{|c|c|c|c|}
\hline & $\begin{array}{c}\text { VINOS } \\
\text { BLANCOS }\end{array}$ & \begin{tabular}{|c|} 
VINOS \\
ROSADOS
\end{tabular} & $\begin{array}{c}\text { VINOS } \\
\text { TINTOS }\end{array}$ \\
\hline \multirow{9}{*}{ ơ } & Amarillo grisáceo & Rosa violáceo & Rojo Violáceo \\
\hline & Amarillo verdoso & Rosa frambuesa & Rojo púrpura \\
\hline & Amarillo paja & Rosa fresa & Rojo granate \\
\hline & Dorado & Rosa grosella & Rojo cereza \\
\hline & Oro viejo & Piel cebolla & Rojo rubí \\
\hline & Ámbar & Salmón & Rojo teja \\
\hline & Castaño & & \\
\hline & Caoba & & \\
\hline & Limpidez & Limpidez & Limpidez \\
\hline \multirow{28}{*}{ 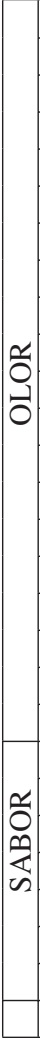 } & Frutos Rojos: & & \\
\hline & Frutos Negros & & \\
\hline & Frutos Blancos & & \\
\hline & Frutos Tropicales & & \\
\hline & Frutos Cítricos & & \\
\hline & Frutos Hueso & & \\
\hline & Frutos Secos & & \\
\hline & Flores Blancas & & \\
\hline & Flores Rojas & & \\
\hline & Flores Azules & & \\
\hline & Vegetales & & \\
\hline & Especies & & \\
\hline & Hierbas Aromáticas & & \\
\hline & Madera & & \\
\hline & Empireumáticos & & \\
\hline & Balsámicos & & \\
\hline & Minerales & & \\
\hline & Animales & & \\
\hline & Microbiológicos & & \\
\hline & Químicos & & \\
\hline & Dulzor & & \\
\hline & Acidez & & \\
\hline & Amargor & & \\
\hline & Salinidad & & \\
\hline & Astringencia & & \\
\hline & Cuerpo/Estructura & & \\
\hline & Persistencia & & \\
\hline & Calidad global & & \\
\hline
\end{tabular}

1 (perceptible), 2 (considerable) y 3 (muy intenso). La evaluación de todos los descriptores la realizó el panel de cata y el valor final fue asignado por consenso.

La estructura del sistema de evaluación de los vinos fue registrada como propiedad intelectual con el nombre de Quanta ${ }^{\circledR}$.

\subsection{Software de educación a distancia}

Se ha utilizado el software libre de código abierto Moodle ver. 2.7. Esta es una aplicación web para producir cursos modulares basados en internet que soporta una moderna pedagogía social basada en un modelo de enseñanza-aprendizaje constructivista (https://moodle.org/). Las evaluaciones sensoriales de los vinos se cargaron en la aplicación como exámenes tipo test de múltiple respuesta, donde cada posible respuesta corresponde a la intensidad de cada atributo. Para la realización de reuniones virtuales y tutoría electrónica 
se ha empleado la aplicación Adobe Connect (Adobe Systems Incorporated; USA).

\subsection{Evaluación de los alumnos}

El aprendizaje del alumno se mide en base a la diferencia de su evaluación con la de los profesores. La ponderación de los atributos de color, olor y sabor se ha basado en la utilizada en la ficha de degustación de vinos de la OIV [16]. De un total de 100 puntos, 15 son distribuidos entre las 5 posibles cuestiones atribuidas a la vista (color dominante e intensidad; color secundario e intensidad; limpidez), 30 a las cuestiones relacionadas con el aroma (intensidad de los 20 descriptores aromáticos empleados); 44 puntos a las relacionadas con el sabor y tacto bucal (intensidad de los 4 sabores básicos, cuerpo, astringencia y persistencia), y 11 puntos a la calidad global del vino. El valor atribuido a cada cuestión individual es asignado en su totalidad cuando la respuesta proporcionada por el alumno es coincidente con la previamente cargada por el profesor. En el caso de un acierto parcial (un color adyacente en la carta de colores, una intensidad adyacente en sabores o en aromas presentes en el vino, se asigna un valor de entre el 25 y el $75 \%$ del total de la pregunta, dependiendo del caso y el número de respuestas posibles. La suma de todos estos valores arroja un valor total máximo de 100 que se transforma luego a una escala entre 0 y 10.

\subsection{Cursos realizados y alumnos inscritos}

Los cursos se han ofertado mediante la plataforma de actividades de extensión universitaria de la Universidad de Cádiz, dentro del programa Enouca de difusión de la cultura del vino (https://celama.uca.es/enouca). Se han desarrollado tres cursos no presenciales entre los años 2012 y 2014 con un total de 106 alumnos inscritos.

\subsection{Tratamiento estadístico de los datos}

Se realizó un detallado análisis estadístico de los resultados obtenidos con el fin de comprobar el aprendizaje de los alumnos mediante la evolución de las calificaciones obtenidas durante el curso. Además, se estudió la influencia de los siguientes factores: género del catador, experiencia previa y tipo de vino.

Los datos no paramétricos y de ordenación se analizaron mediante estudio de varianza para tamaño de muestras desiguales, utilizando para ello la prueba $\mathrm{H}$ de Kruskal y Wallis. Se calcularon las comparaciones post hoc mediante la prueba de aproximación de Chi Cuadrado. Los datos paramétricos se analizaron mediante análisis de varianza (ANOVA) de un factor y de varios factores con interacción El nivel mínimo de significación utilizado en todos los análisis estadísticos fue del $5 \%$.

El tratamiento inicial de los datos para la obtención de frecuencias, porcentajes, medias, desviaciones estándar y rangos se realizó mediante Microsoft Excel $2013^{\circledR}$.

Los análisis estadísticos se realizaron mediante los sofwares Statistix 8.0 (NH Analytical Software, Roseville, MN; USA) y Statgraphics Centurion versión 16.2.04 (Statpoint Technologies; USA).

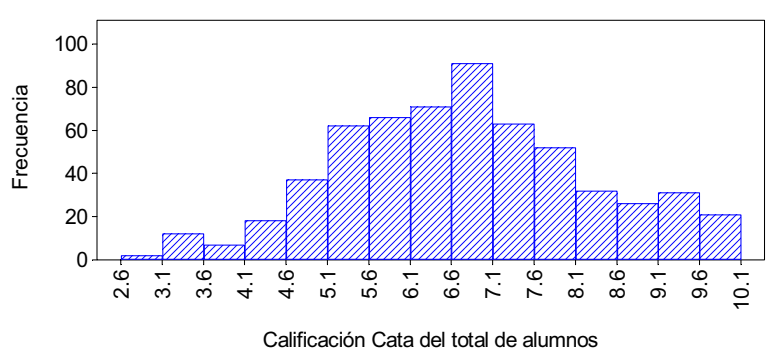

Figura 1. Histograma de frecuencias de las calificaciones.
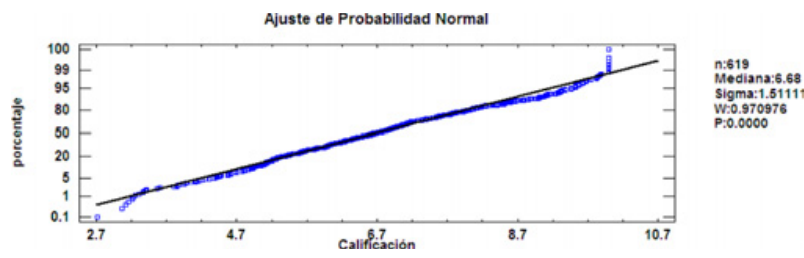

Figura 2. Gráfico de distribución normal.

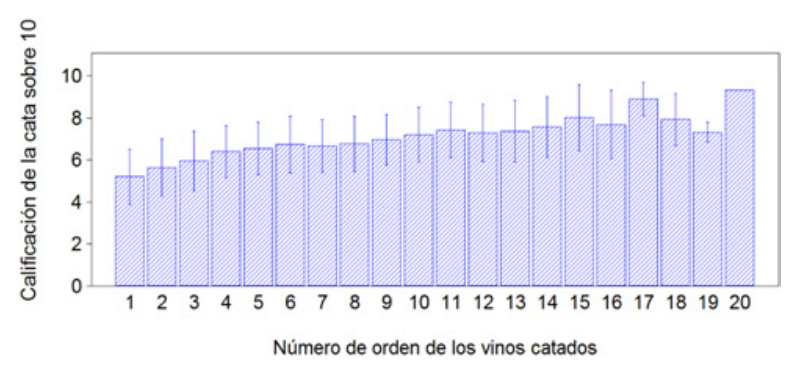

Figura 3. Evolución de las calificaciones según el orden en que se ha realizado la cata.

\section{Resultados y discusión}

\subsection{Distribución de frecuencias en las calificaciones}

En primer lugar se ha comprobado que las calificaciones presentan una distribución normal, condición necesaria para algunos de los análisis de varianza posteriores. Para ello, se ha realizado un histograma de frecuencias y un gráfico de probabilidad normal que se presentan en las Figs. 1 y 2.

En ellas se pueden comprobar que las calificaciones obtenidas por los alumnos presentan una distribución normal o gaussiana.

\subsection{Aprendizaje de los alumnos}

La evaluación del aprendizaje de los alumnos se ha realizado a partir de las calificaciones obtenidas a lo largo de las sucesivas sesiones de cata de vinos realizadas por ellos. Sobre estos datos se realizó la prueba $\mathrm{H}$ de Kruskal Wallis One-Way Nonparametric, siendo la variable dependiente la calificación y la independiente el orden de realización de la cata. En la Fig. 3 se han representado las calificaciones medias y desviaciones de cada sesión de cata. En ella se puede apreciar que las calificaciones medias superan el 5 (aprobado) desde el principio y presentan un aumento constante a lo largo del curso, presentando las calificaciones finales un valor medio en torno a 7,5-8. 
Tabla 2. Tabla ANOVA para Calificación por Género.

\begin{tabular}{|c|c|c|c|c|}
\hline Fuente & $\begin{array}{c}\text { Suma de } \\
\text { Cuadrados }\end{array}$ & $\begin{array}{c}\text { Cuadrado } \\
\text { Medio }\end{array}$ & Razón-F & Valor-P \\
\hline Entre grupos & 15.0922 & 15.0922 & 6,73 & 0,0095 \\
\hline Intra grupos & 1384.62 & 2.24412 & & \\
\hline Total & 1399.71 & & & \\
\hline
\end{tabular}

Valores medios de calificación por género y LSD de Fisher

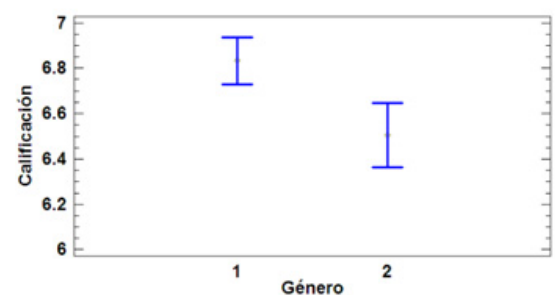

Figura 4. Valores medios y LSD de las calificaciones por género.

El test de Kruskal-Wallis mostró el valor muy elevado del estadístico de 170,652, con una $\mathrm{p}<0,001$, lo que indica que la diferenciación de valores de las calificaciones durante las sucesivas sesiones de cata realizadas es muy significativa.

\subsection{Diferenciación por género}

De los 106 alumnos que realizaron el curso un 64,8\% eran mujeres y un $35,2 \%$ hombres. Se ha realizado un análisis de varianza ANOVA con la variable dependiente calificación y la independiente género, obteniéndose los valores de la Tabla 2.

La tabla ANOVA descompone la varianza de Calificación en dos componentes: un componente entregrupos y un componente dentro-de-grupos. La razón-F, que en este caso es igual a 6,73, es el cociente entre el estimado entre-grupos y el estimado dentro de grupos. Puesto que el valor-P de la prueba-F es menor que 0,05 , existe una diferencia estadísticamente significativa entre la media de Calificación entre un nivel de género y otro, con un nivel del $95.0 \%$ de confianza.

Esta afirmación es confirmada en la Fig. 4 donde se representan los valores medios y LSD (Least significant difference) por género, siendo 1la mujer y 2 el hombre.

Estos resultados presentan una concordancia con estudios recientes desde una perspectiva fisiológica, donde se encuentran diferencias sensoriales basadas en el género (15).

De todas formas, debe tenerse en cuenta que el número de mujeres y hombres que realizaron el curso no estaba equilibrado.

\subsection{Diferenciación por conocimientos previos}

Los conocimientos previos de los alumnos en análisis sensorial de vinos fueron autoevaluados previamente por los alumnos como 0 (sin experiencia), 1 (experiencia corta), 2 (experiencia media) y 3 (experiencia amplia).

Sobre estos resultados se realizó un análisis de varianza ANOVA de un factor, obteniendo los resultados de la Tabla 3 y la Fig. 5.
Tabla 3. Tabla ANOVA para Calificación por Experiencia.

\begin{tabular}{|c|c|c|c|c|}
\hline Fuente & $\begin{array}{c}\text { Suma de } \\
\text { Cuadrados }\end{array}$ & $\begin{array}{c}\text { Cuadrado } \\
\text { Medio }\end{array}$ & Razón-F & Valor-P \\
\hline Entre grupos & 230.97 & 76.9901 & 40.51 & 0.0000 \\
\hline Intra grupos & 1168.74 & 1.90039 & & \\
\hline Total & 1399.71 & & & \\
\hline
\end{tabular}

Calificaciones medias y LSD Fisher según experiencia

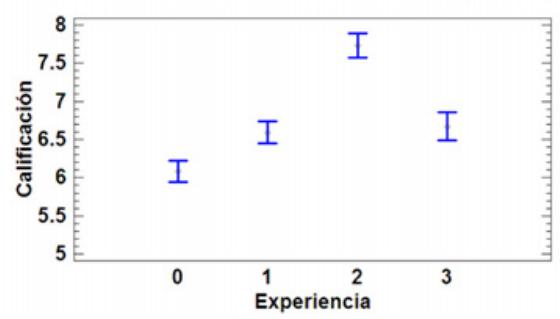

Figura 5. Calificaciones de catadores con diferente experiencia inicial.

Interacción Género-Experiencia

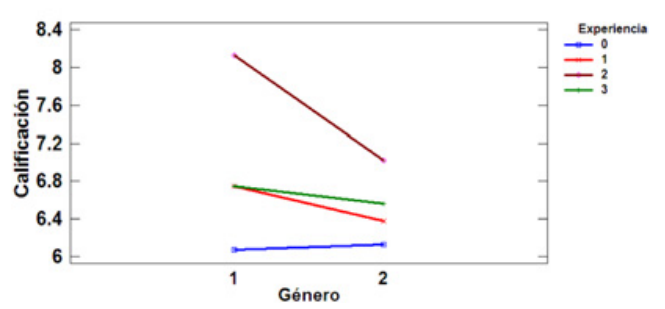

Figura 6. Efecto de la interacción Experiencia y Género.

El valor elevado de la razón-F, que en este caso es igual a 40,51 y el valor-P de la prueba menor que 0,05 , indican que existe una diferencia estadísticamente significativa de la media de Calificación entre un nivel de Experiencia y otro, con un nivel del $95,0 \%$ de confianza. Pero sorprendentemente son los alumnos con nivel de experiencia declarada de 2 los que obtienen mayores calificaciones. Independientemente de la fiabilidad de la declaración previa de experiencia realizada por los alumnos, este hecho puede ser debido a un factor de "confianza" por los catadores más expertos que les hace reducir su nivel de concentración. Este hecho ha sido repetidamente observado por los autores en ocasiones en que catadores expertos han catado junto a catadores menos expertos y han sido estos últimos los que han obtenidos mejores resultados en sus apreciaciones.

Si se analiza el efecto diferenciado de la experiencia en las calificaciones según los géneros en la Fig. 6 se observa que el efecto de inversión del orden previsible se produce en mayor medida en las mujeres que en los hombres.

\subsection{Diferenciación por tipos de vino}

Los vinos se han clasificado en cinco tipos: blancos (1), rosados (2), tintos jóvenes (3), tintos con crianza (4) y generosos (5). Se ha realizado un análisis de varianza ANOVA sobre las calificaciones obtenidas en los diferentes tipos para saber si existen diferencias 
Tabla 4. Tabla ANOVA para Calificación por Tipo de vino.

\begin{tabular}{|c|c|c|c|c|}
\hline Fuente & $\begin{array}{c}\text { Suma de } \\
\text { Cuadrados }\end{array}$ & $\begin{array}{c}\text { Cuadrado } \\
\text { Medio }\end{array}$ & Razón-F & Valor-P \\
\hline Entre grupos & 45.1943 & 11.2986 & 5,12 & 0,0005 \\
\hline Intra grupos & 1354.52 & 2.20606 & & \\
\hline Total & 1399.71 & & & \\
\hline
\end{tabular}

Calificaciones medias y LSD Fisher

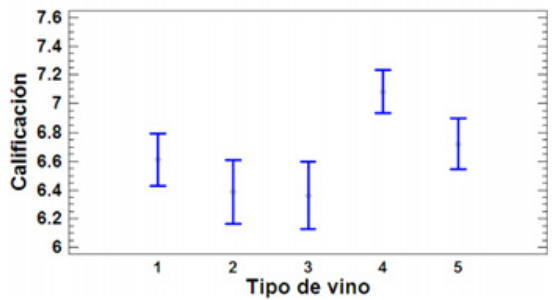

Figura 7. Calificaciones medias en los diferentes tipos de vino.

significativas. Los resultados de la Tabla 4 y Fig. 7 indican que los vinos tintos con crianza obtienen una nota diferenciada y significativamente superior a la de los otros vinos. Asimismo, los vinos generosos también presentan unas calificaciones ligeramente superiores a las de los restantes tipos de vino.

\subsection{Encuesta de satisfacción}

Al finalizar el curso, se pidió a los alumnos que hicieran una encuesta de satisfacción anónima para conocer sus opiniones sobre el desarrollo del curso. Cada concepto está evaluado de 0 (lo peor) a 10 (lo mejor). Sus resultados medios se presentan en la Tabla 5 .

Como se puede apreciar, la valoración global de 8,67 puede calificarse de excelente. De los conceptos valorados cabe destacar en sentido positivo las opiniones sobre la utilidad del campus virtual, la ficha de cata organizada como un examen tipo test, el contenido general del curso, las tutorías electrónicas y las respuestas a las preguntas realizadas. En sentido negativo cabe destacar la menor aceptación que mostró la utilidad de la carta de colores cuyo diseño debe ser mejorado en relación a los colores impresos.

\section{Conclusiones}

El sistema de enseñanza-aprendizaje desarrollado basado en e-learning se ha mostrado eficaz y ha permitido el aprendizaje a distancia del análisis sensorial de vinos con una eficacia estadísticamente significativa.

Con estos estudios se ha obtenido información sobre las percepciones de los participantes, una vez superada la fase de adiestramiento, respecto a la cata de los diferentes vinos comerciales catados, que alcanzaron un número de 60 referencias diferentes en cada una de las ediciones realizadas.

Se ha observado una diferenciación significativa de los resultados obtenidos, relacionada con el género, con
Tabla 5. Encuesta de satisfacción de los alumnos.

\begin{tabular}{|l|l|l|}
\hline CONCEPTO & MEDIA & D.E. \\
\hline ADIESTRAMIENTO DE LOS SENTIDOS & & \\
\hline Adiestramiento de la vista & 8,34 & 1,10 \\
\hline Adiestramiento del olfato (preparadas en casa) & 7,46 & 1,76 \\
\hline Adiestramiento del olfato (frascos de aromas) & 7,83 & 1,75 \\
\hline Adiestramiento del gusto & 8,38 & 0,99 \\
\hline Adiestramiento del tacto & 8,04 & 1,12 \\
\hline METODOLOGÍA DE CATA DE VINOS & & \\
\hline Evaluación del color mediante carta de colores & 7,60 & 1,72 \\
\hline Evaluación del aroma & 8,52 & 1,27 \\
\hline Evaluación del sabor y tacto bucal & 8,20 & 0,98 \\
\hline Utilidad de la ficha de cata & 8,76 & 1,07 \\
\hline Utilidad del campus virtual & 8,84 & 0,92 \\
\hline SOBRE LOS CONTENIDOS & & \\
\hline Las grabaciones realizadas ex-profeso & 8,56 & 1,27 \\
\hline Otras grabaciones utilizadas & 8,14 & 1,10 \\
\hline Materiales facilitados (carta de colores) & 7,60 & 1,62 \\
\hline Materiales facilitados (frascos de aromas) & 7,98 & 2,06 \\
\hline Número de botellas a catar sugeridas & 8,63 & 1,44 \\
\hline Contenido general del curso & 8,64 & 1,47 \\
\hline ORGANIZACIÓN Y ATENCIONES & & \\
\hline Duración & 8,35 & 1,34 \\
\hline Tutorías & 8,80 & 1,12 \\
\hline Respuestas a las dudas & 9,18 & 1,15 \\
\hline VALORACIÓN GLOGAL & 8,67 & 0,94 \\
\hline D.E. Dín
\end{tabular}

D.E. Desviación estándar.

la experiencia previa de los participantes y con el tipo de vino. Se ha observado que las mujeres han obtenido mejores calificaciones que los hombres, que los catadores que tenían una experiencia previa media han obtenido mejores calificaciones que los de mayor experiencia y por último que los vinos mejor calificados han sido los tintos con crianza.

Los autores agradecen la esponsorización y ayuda del Campus Excelencia Internacional Agroalimentario (CEIA3), así como el soporte facilitado por el Vicerrectorado de Proyección Internacional y Cultural de la Universidad de Cádiz.

\section{Referencias}

[1] J. Cabero Almenara. "Bases pedagógicas del $e$ learning". Revista de Universidad y Sociedad del Conocimiento. 3, 1,1-10,(2006)

[2] J. G. Ruiz, M.J. Mintzer, R.M. Leipzig,.The Impact of e-Learning in Medical Education Academic Medicine,81,3, 207-212,(2006)

[3] A. Jubert, C. Pogliani, A. M. Tocci, A. Vallejo. Chemistry E-course for first year engineering students. EducaciónQuímica, 23, 1,16-22, (2012)

[4] S. Fincke, H. D, Wuttke . E-learning in engineering studies - experience of the Ilmenau University of Technology. Biomed Tech, 58, 1 DOI 10.1515/bmt2013-4415 (2013)

[5] University of California, Davis. Winemaking certificate program. http://extension.ucdavis.edu/ 
unit/winemaking/certificate/winemaking/. Last accessed $4^{\text {th }}$ August 2014

[6] INEA. Escuela Universitaria de Ingeniería Agrícola. Universidad de Valladolid (España). Curso de Análisis sensorial y cata de vinos. http:// www . inea . org/index. php?option=com_content \&view=article\&id=161\&Itemid=1244\#arriba. Lastaccessed $4^{\text {th }}$ August 2014

[7] D. C. Taylor, T. H. Dodd, N. Barber. Impact of Wine Education on Developing Knowledge and Preferences: An Exploratory Study. Journal of WineResearch, 19,3 http://www.tandfon line.com/toc/cjwr20/19/3, 193-207, (2008)

[8] ISO 8586:2012. Sensory analysis. General guidelines for the selection, training and monitoring of selected assessors and expert sensory assessors

[9] Y Cadot, S. Caillé, A. Samson, G. Barbeau, V. Cheynier. Sensory dimension of wine typicality related to a terroir by Quantitative Descriptive Analysis, Just About Right analysis and typicality assessment. Analytica Chimica Acta 660 53-62, (2010)

[10] S. Mirarefi, S.D. Menke, S.-Y. Leevol. Sensory Profiling of Chardonel Wine by Descriptive Analysis. J. Food Science, 69, 6, 211-217, (2004)
[11] A. Vannier, O.X. Bruna, M.H. Feinberg. Application of sensory analysis to champagne wine characterisation and discrimination. Food Quality and Preference, 10, 101-107, (1999)

[12] L. Perrin, R. Symoneaux, I. Maître, C. Asselin, F. Jourjon, J. Pagès. Comparison of three sensory methods for use with the Napping procedure: Case of ten wines from Loire valley. Food Quality and Preference, 19, 1-11, (2008)

[13] J. M. Murray, C. M. Delahunty, I. A. Baxter. Descriptive sensory analysis: past, present and future. Food Research International, 34, 461-471, (2001)

[14] M. Vilanova, Sensory desdriptive analysis and consumer acceptability of godello wines from Valdeorras appellation origen controlee (North Spain) Journal of Sensory Studies, 21, 362-372 (2006)

[15] L. A. da Silva, S.M. Lin, M.J. Teixeira, JTT de Siqueira, W Jacob Filho, SRDT de Siqueira. Sensorial differences according to sex and ages. Oral Diseases 20, e103-e110 (2014)

[16] OIV, Resolución OIV/CONCOURS 332A/2009. Norma OIV de los concursos internacionales de vinos y bebidas espirituosas de origen vitivinícola 